\title{
Bose-fermi Equivalence and Interacting String Field Theory
}

A Abdurrahman F Anton and J Bordes

May 1995 


\section{(C) Council for the Central Laboratory of the Research Councils 1995}

Enquiries about copyright, reproduction and requests for additional copies of this report should be addressed to:

The Central Laboratory for the Research Councils

Library and Information Services

Rutherford Appleton Laboratory

Chilton

Didcot

Oxfordshire

OX11 OQX

Tel: 01235445384 Fax: 01235446403

E-mail library@rl.ac.uk

ISSN 1358-6254

Neither the Council nor the Laboratory accept any responsibility for loss or damage arising from the use of information contained in any of their reports or in any communication about their tests or investigations. 


\title{
BOSE-FERMI EQUIVALENCE AND INTERACTING STRING FIELD THEORY
}

\author{
A. Abdurrahman ${ }^{1}$, F. Anton ${ }^{2}$, J. Bordes ${ }^{3}$ \\ Department of Particle Physics \\ Rutherford Appelton Laboratory \\ Chilton DIDCOT, Oxon OX11 OQX, U.K. \\ U.K.
}

\begin{abstract}
We show that the Bosonic and the Fermionic realization of the ghost vertex in the Half-String (HS) Operator approach to Witten's String Field Theory (WSFT) are equivalent. In the process we discover that higher vertices (i.e., $N>3$ ) can be eliminated in WSFT using only the overlap conditions defining the interaction vertex and ghost number counting.
\end{abstract}

May 1995

\footnotetext{
1'E-Mail: abdurrahman@v2.rl.ac.uk

${ }^{2}$ Department of Physics, University of Oxford, 1 Keble Road, Oxford, OX1 3NP, U.K.

${ }^{3}$ Departament de Física Teórica, Universitat de Valencia, Dr. Moliner 50, E-46100, Burjassot, Spain and IFIC, Centro Mixto Universitat de Valencia-CSIC, Spain.
} 
The Half-String Formulation (HSF) of interacting string field theory, originally, motivated by Witten in [1] and formulated in detail in $[2,4,5,6]$ turn out to be most efficient when computing interaction vertices of the theory and when discussing the invariance and potential anomalies of the theory due to choosing a preferred point $\left(\sigma=\frac{\pi}{2}\right)$ in Witten's String Field Theory. There is now a considerable body of work on the HSF of interacting string field theory of both open and closed bosonic string $[2,4,5,6,7]$.

In the HSF, one singles out the mid-point of the string thus breaking the string into two pieces, $\chi^{(r)}(\sigma)\left(\varphi^{(r)}(\sigma)\right)$, with $r=1,2$ referring to the left-right parts of the string, of the orbital (ghost) coordinate of the string . The string functional $\psi$ is, then, a functional $\psi\left[\chi^{(1)}(\sigma), \chi^{(2)}(\sigma), X\left(\frac{\pi}{2}\right), \varphi^{(1)}(\sigma), \varphi^{(2)}(\sigma), \phi\left(\frac{\pi}{2}\right)\right]$ of $\chi^{(1)}(\sigma), \chi^{(2)}(\sigma)$ and $X\left(\frac{\pi}{2}\right)^{4}$ (and the corresponding ghost coordinates). We have seen, in $[3,4,5,6]$, that in this formulation the basic operations of $W S F T$, the star product $(*)$ and integration $(f)$ become matrix multiplication (.) and trace $(T r)$ respectively and all the axioms of WSFT are seen to be satisfied. In this formulation, one has a map $F_{s}^{x}\left(F_{s}^{\phi}\right) \longrightarrow H_{s}^{\chi}\left(H_{s}^{\varphi}\right)$; where $F_{s}^{x}\left(F_{s}^{\phi}\right)$ is the complete set of the FS oscillator modes $\left\{x_{0}, x_{1}, x_{2}, \ldots, p_{0}, p_{1}, p_{2}, \ldots\right\}\left(\left\{\phi_{0}, \phi_{1}, \phi_{2}, \ldots, p_{0}^{\phi}\right.\right.$, $\left.\left.p_{1}^{\phi}, p_{2}^{\phi}, \ldots\right\}\right)$ and $H_{s}^{\chi}\left(H_{s}^{\varphi}\right)$ is the complete set of the HS oscillator modes $\left\{X\left(\frac{\pi}{2}\right), \chi_{1}^{(r)}\right.$, $\left.\chi_{2}^{(r)}, \ldots, P_{\frac{\pi}{2}}, p_{1}^{(r)}, p_{2}^{(r)}, \ldots ; r=1,2\right\}\left(\left\{\phi\left(\frac{\pi}{2}\right), \varphi_{1}^{(r)}, \varphi_{2}^{(r)}, \ldots, P_{\frac{\pi}{2}}^{\phi}, p_{1}^{\varphi^{(r)}}, p_{2}^{\varphi^{(r)}}, \ldots ; r=1,2\right\}\right)$. This map follows from the definition of the HS coordinates

$$
\chi^{(r)}(\sigma)=-\left\{\begin{array}{l}
X(\sigma)-X\left(\frac{\pi}{2}\right), \text { if } r=1, \\
X(\pi-\sigma)-X\left(\frac{\pi}{2}\right), \text { if } r=2 ; \quad \sigma \in\left[0, \frac{\pi}{2}\right]
\end{array}\right.
$$

(with $\varphi^{(r)}(\sigma)$ being given by the same definition with $X \longrightarrow \phi$ ) and it has been constructed in $[4,5]$ and is given by an infinite dimensional nonsingular matrix.

In [5], hereinafter referred to as (I), the ghost sector of the theory was constructed using the bosonic formulation as originally presented by Witten [1]. There, the precise form of the ingredients of the theory, were constructed in terms of HS (first quantized) ghost Fock space states and operators. And there was established that the FS ghost Fock space obtained by quantizing the full open string is given by a tensor product of the two HS Fock spaces and a Fock space of functions of the mid-point $\phi\left(\frac{\pi}{2}\right)$.

In the present paper we wish to establish the bose-fermi equivalence, which was not proved at all in (I), in particular, we want to emphasize the role played

\footnotetext{
${ }^{4}$ Another approach in which the mid-point plays a central role has been discussed in ref. [8], although in a different context.
} 
by the ghost insertion factor $e^{i Q_{\alpha} \phi\left(\frac{\pi}{2}\right)}$ in the HSF of WSFT ${ }^{5}$. We will see, in the proof of the bose-fermi equivalence in the HSF to be given below, that the ghost insertion play a crucial role. Some of the results presented here are obtained in the same way as in [5]. Although some computational details are relegated to the appendix, this paper is not meant to be self-contained in the sense that we rely on (I) for notation and indeed for many other details only alluded to below. 1. The $N$-Vertex

In the Half String approach to string field theory the elements of the theory are defined by $\delta$ - function-type overlaps

$$
V_{N}^{\varphi}=e^{i \sum_{\mu=1}^{N} Q_{\mu}^{\varphi} \phi\left(\frac{\pi}{2}\right)} V_{N, 0}^{\varphi}
$$

with

$$
V_{N, 0}^{\varphi}=\prod_{\mu=1}^{N} \prod_{\sigma=0}^{\frac{\pi}{2}} \delta\left(\varphi_{\mu}^{(1)}(\sigma)-\varphi_{\mu-1}^{(2)}(\sigma)\right)
$$

The index $\mu$ refers to the $\mu$ th string (it is understood that $\mu-1=0 \equiv N$ ) and the 1 and 2 superscripts refer to left and right halves of the string respectively. The $Q^{\varphi}$ is the ghost number insertion at the mid-point which is needed for the BRST invariance of the theory $[1,9,10,6]$. In the Hilbert space of the theory the $\delta$ functions translate into operator overlap equations which determine the precise form of the vertex. The vertex will be given by exponentials of quadratic form in the HS creation operators. However, unlike the FS approach $[11,12,10]$ where determining the exact form of the vertex involves using Neumann functions and (involved) conformal mapping techniques, which are based on the methods in [14], here the exact form of the vertex can be read directly from the overlap conditions defining the HS ghost coordinates

$$
\left|V_{N, 0}^{\varphi}\right\rangle=e^{-\frac{1}{2} b_{\mu,-n}^{(r)} \Theta_{\mu n, \nu m}^{r, s} b_{\nu,-m}^{(s)}} \prod_{\mu=1}^{N}\left|0>_{\mu}^{(1)}\right| 0>_{\mu}^{(2)}
$$

where the matrix $\Theta$ is given by

$$
\Theta_{\mu n, \nu m}^{r, s}=\left(\delta^{r+1, s} \delta_{\mu-1, \nu}+\delta^{s+1, r} \delta_{\nu-1, \mu}\right) \delta_{n m}
$$

\footnotetext{
${ }^{5}$ For more details we refer the reader to the original work of Witten [1].
} 
It is worth noting that $\Theta=\Theta^{T}$. At this point it becomes clear that the HS approach is most useful when extended to closed string field theory (CSFT) [7]. There one needs to construct the exact form of the vertex for $N=1,2,3, \ldots, \infty$ in order to write down the closed string action.

To emphasize the simplicity of the HS approach it is instructive to derive (4) from first principles. The $\delta$ - functions defining the HS vertices, in the Hilbert space of the theory, translate to the overlap constraints

$$
\left[\varphi_{\mu}^{(1)}(\sigma)-\varphi_{\mu}^{(2)}(\sigma)\right] \mid V_{N, 0}^{\varphi}>=0, \quad \sigma \in\left[0, \frac{\pi}{2}\right]
$$

where the $\varphi_{\mu}^{(r)}(\sigma) ; r=1,2$; are the HS ghost coordinate defined in (I). The mode expansion of $\varphi_{\mu}^{(r)}(\sigma)$ in the HS formulation is given by

$$
\varphi_{\mu}^{(r)}(\sigma)=\sqrt{2} \sum_{n \geq 1} \varphi_{\mu n}^{(r)} \cos (2 n-1) \sigma
$$

where $\varphi_{\mu n}^{(r)}$ are canonical ghost coordinates with canonical momenta $p_{\mu n}^{(r)}=$ $-i \frac{\partial}{\partial \varphi_{\mu n}^{(r)}}$. Thus in terms of HS oscillator modes the overlap conditions read

$$
\left[\varphi_{\mu n}^{(1)}-\varphi_{\mu n}^{(2)}\right] \mid V_{N, 0}^{\varphi}>=0 ; n=1,2, \ldots, \infty ; \mu=1,2, \ldots, N
$$

Introducing the HS creation and annihilation operators

$$
b_{\mu n}^{(r)}=-\frac{i}{\sqrt{2}}\left(\frac{2 n-1}{2}\right)\left(\varphi_{\mu n}^{(r)}+\frac{2 i}{2 n-1} p_{\mu n}^{(r)}\right)
$$

(with $b_{\mu-n}^{(r)} \equiv b_{\mu n}^{(r) \dagger}$ ) and using equations (3) and (7) we arrive at $(4)^{6}$.

\section{The Proof of Equivalence}

Our task now is to show that the bosonizied HS ghost vertices, (1), satisfy the $c-$ and $b$-overlap equations displayed below. To carry out the proof we will utilize the bosonization formulas

$$
c_{+}(\sigma)=: e^{i \phi_{+}(\sigma)}:, \quad b_{+}(\sigma)=: e^{-i \phi_{-}(\sigma)}:
$$

\footnotetext{
${ }^{6}$ It is important to notice that (5) gives only half the constraints on the Matrix $\Theta$ the other half of the constraints is given by the analogous equations for $p_{\mu}^{(r)}(\sigma)$.
} 
(with $c_{-}(\sigma)=c_{+}(-\sigma)$, and $b_{-}(\sigma)=b_{-}(-\sigma)$ ). However, in the bosonization of the fermionic coordinates, using the standard procedure (see ref. 17 in [15]), it is not obvious that all ingredients of the theory employing the bosonic field $\phi(\sigma)$ are equivalent to those constructed using the original fermi fields appearing in the left hand side of the above relations ${ }^{7}$. It is the purpose of this letter to establish this equivalence. We will see that the ghost vertices in the HS operator formulation obey the same overlap equations as the fermionic vertices and are in fact identical. Consequently one is free to use either formulation of the ghost sector of the theory. We have seen, for example, that when discussing the $K_{n}$ invariance of the theory and computing vertices to all orders the bosonic formulation was employed $[12,5]$ while when discussing the BRST invariance of the theory it was easier to employ the fermionic formulation $[9,10,6]$.

To prove the Bose Fermi equivalence we have to show that both realizations of the vertex satisfy the same overlap equations. This can be carried out using the above bosonized formulas. Now the comparison with the FS formulation of WSFT employed in $[12,9,10]$ is of utmost importance, so now we pause to discuss this briefly. In the FS approach to WSFT the complexity arises because of the complicated scattering geometry involved in the construction of the 3-Vertex. On the other hand, in the HS formulation, the situation is very simple due to the simple form of the matrix $\Theta$ defining the $N$-Vertex. It is also useful to note that the proof of the $c$-overlaps on the bosonic realization of the vertex is essentially identical to the proof of the $\varphi$-overlaps ${ }^{8}$ given in (I). This is because the fermionic ghost field $c(\sigma)$ is an exponential of the bosonic ghost $\phi(\sigma)$ up to an infinite ordering factor. An immediate consequence is that the operator part of the $c$-overlaps and the $b$-overlaps will hold for all $\sigma$ as we shall see. However, normal ordering introduces a phase factor when $c$ ghost field or $b$ ghost field acts on the bosonic ghost vertex. Therefore it is crucial for the equivalence of the two realizations of the vertex that the phase factors obey the overlap equations.

The fermionic ghost overlap equations are

\footnotetext{
${ }^{7}$ One has to remember that the equality signs appearing in the above equations are misleading, they are to be viewed as relationships that have to be verified at the end of the carried program.

${ }^{8}$ The proof of the $\varphi$-overlaps on the bosonic realization of the vertex is identical to the derivation of the ghost vertices given at the beginning of the letter.
} 


$$
\begin{gathered}
c_{\mu}(\sigma)=- \begin{cases}c_{\mu-1}(\pi-\sigma), & \sigma \in\left[0, \frac{\pi}{2}\right] \\
c_{\mu+1}(\pi-\sigma), & \sigma \in\left[\frac{\pi}{2}, \pi\right]\end{cases} \\
b_{\mu}(\sigma)= \begin{cases}b_{\mu-1}(\pi-\sigma), & \sigma \in\left[0, \frac{\pi}{2}\right] \\
b_{\mu+1}(\pi-\sigma), & \sigma \in\left[\frac{\pi}{2}, \pi\right]\end{cases}
\end{gathered}
$$

Note that the $c$ equation is like the momentum overlap and the $b$ equation is like the coordinate overlap. To show that the HS vertices given in (1) obey the above overlap equations we first express the fermionic ghost fields in terms of the bosonizied HS ghost coordinates. Using the bosonizied formula and the results of (I) we have

$$
\begin{aligned}
c_{\mu}(\sigma)= & e^{-\frac{2}{\pi} \sum_{n=1}^{\infty} \frac{1}{(2 n-1)^{3}}} e^{i \phi_{\mu}\left(\frac{\pi}{2}\right)} e^{i \frac{\sigma}{2}} e^{\left(D+D^{*}+C+\frac{2}{\pi^{2}} \sum_{n=1}^{\infty} \frac{1}{(2 n-1)} \partial_{\sigma, n}^{*} \sum_{n}^{c}(\sigma)\right)} \\
& \times e^{\left(\alpha_{n}^{r} b_{n \mu}^{(r)}-\alpha_{n}^{(r) *} b_{-n \mu}^{(r)}\right)} e^{\left(i \sigma+\sum_{n=1}^{\infty} \frac{(-)^{n}}{2 n}\left(e^{2 i n \sigma}-e^{-2 i n \sigma}\right)\right) P_{\mu}^{\varphi}}
\end{aligned}
$$

(where $\phi_{\mu}\left(\frac{\pi}{2}\right)$ is the mid-point ghost coordinate with canonical momenta, $P_{\mu}^{\varphi}=$ $\left.-i \frac{\partial}{\partial \phi_{\mu}\left(\frac{\pi}{2}\right)}\right)$. The various quantities $D, D^{*}, \ldots$ appearing in the above expression are given by

$$
\begin{gathered}
D(\sigma)=\frac{1}{2} \sum_{m=1}^{\infty} \frac{1}{2 m-1} \sum_{n=1}^{\infty} e^{-2 i n \sigma} A_{2 m-12 n} \sum_{k=1}^{\infty} e^{-2 i k \sigma} S_{2 m-12 k} \\
C(\sigma)=-\sum_{m=1}^{\infty} \frac{1}{2 m-1} \sum_{n=1}^{\infty} e^{2 i n \sigma} S_{2 m-12 n} \sum_{k=1}^{\infty} e^{-2 i k \sigma} S_{2 m-12 k} \\
\partial_{\sigma, n}=1+\frac{i}{2 n-1} \partial_{\sigma}, \quad \Sigma_{m}^{c}(\sigma)=\sum_{n=1}^{\infty} \frac{(-)^{n} \cos n(2 \sigma)}{n^{2}-\left(\frac{2 m-1}{2}\right)^{2}} \\
\alpha_{n}^{r}=\frac{1}{\sqrt{2}}\left(\frac{2}{\pi} \frac{(-)^{n}}{(2 n-1)^{3 / 2}}-\frac{(-)^{n}}{\pi}(2 n-1)^{1 / 2} \partial_{\sigma, n} \Sigma_{n}^{c}(\sigma)-\frac{e^{-i(2 n-1) \sigma}}{(2 n-1)^{1 / 2}}(-)^{r+1}\right)
\end{gathered}
$$

with

$$
A_{2 m-12 n}=B_{2 m-12 n}-B_{2 n 2 m-1}, \quad S_{2 m-12 n}=B_{2 m-12 n}+B_{2 n 2 m-1}
$$

where

$$
B_{n, m}=\frac{(-)^{(n+m+1) / 2}}{\pi}\left(\frac{2 m}{m^{2}-n^{2}}\right)
$$


is the change of representation matrix between the FS and HS ghost coordinates. Notice that this matrix has already appeared in $[4,5,6,2,7]$ for both open and closed bosonic string.

\section{The 1-Vertex}

To establish the bose fermi equivalence we, first, consider the identity operator $\left|I^{\varphi}\right\rangle$. This is very crucial for the fact that the identity operator is a Fock space realization of the integration on the space of string functionals. Setting $N=1$ in (1) we have

$$
\left|I^{\varphi}>=e^{-\frac{3}{2} i \phi\left(\frac{\pi}{2}\right)} e^{-\frac{1}{2} b_{-n}^{(r)} \Theta_{n, m}^{r, s} b_{-m}^{(s)}}\right| 0>^{(1)} \mid 0>^{(2)}
$$

where the factor $e^{-\frac{3}{2} i \phi\left(\frac{\pi}{2}\right)}$ is the ghost insertion at the mid-point of the string that appears in the definition of $\int$ in WSFT (see ref. [1]). The overlap equations for $c$ and $b$ in the case of $I^{c}$ are given by ${ }^{9}$

$$
c(\sigma)=-c(\pi-\sigma)
$$

and

$$
b(\sigma)=b(\pi-\sigma)
$$

respectively. Again using the bosonized formulas and the results obtained in (I), for a single string in the HS formulation, $c(\sigma)$ and $b(\sigma) \operatorname{read}^{10}$

$$
\begin{aligned}
c(\sigma)= & e^{-\frac{2}{\pi} \sum_{n=1}^{\infty} \frac{1}{(2 n-1)^{3}}} e^{i \phi\left(\frac{\pi}{2}\right)} e^{i \frac{\sigma}{2}} e^{\left(D+D^{*}+C+\frac{2}{\pi^{2}} \sum_{n=1}^{\infty} \frac{1}{(2 n-1)} \partial_{\sigma, n}^{*} \Sigma_{n}^{c}(\sigma)\right)} \\
& \times e^{\left(\alpha_{n}^{r} b_{n}^{(r)}-\alpha_{n}^{(r) *} b_{-n}^{(r)}\right)} e^{\left(i \sigma+\sum_{n=1}^{\infty} \frac{(-)^{n}}{2 n}\left(e^{2 i n \sigma}-e^{-2 i n \sigma}\right)\right) P^{\varphi}}
\end{aligned}
$$

and

$$
b(\sigma)=e^{-\frac{2}{\pi} \sum_{n=1}^{\infty} \frac{1}{(2 n-1)^{3}}} e^{-i \phi\left(\frac{\pi}{2}\right)} e^{i \frac{\sigma}{2}} e^{\left(D+D^{*}+C+\frac{2}{\pi^{2}} \sum_{n=1}^{\infty} \frac{1}{(2 n-1)} \partial_{\sigma, n} \Sigma_{n}^{c}(\sigma)\right)}
$$

\footnotetext{
${ }^{9} I^{c}$ is the fermionic ghost identity vertex. Therefore to prove that $I^{\varphi}$ is equivalent to $I^{c}$ we have to show that $I^{\varphi}$ obey the same overlap equations obeyed by $I^{c}$.

${ }^{10}$ Notice that $c(\sigma)(b(\sigma))$ is the same as $c_{+}\left(b_{-}\right)$for $0 \leq \sigma \leq \pi$ and the same as $c_{-}\left(b_{-}\right)$for $-\pi \leq \sigma \leq 0$
} 


$$
\times e^{\left(-\alpha_{n}^{r^{*}} b_{n}^{(r)}+\alpha_{n}^{r} b_{-n}^{(r)}\right)} e^{\left(i \sigma+\sum_{n=1}^{\infty} \frac{(-)^{n}}{2 n}\left(e^{2 i n \sigma}-e^{-2 i n \sigma}\right)\right) P^{\varphi}}
$$

Let us first consider the action of the $c(\sigma)$ fermi field on $\left|I^{\varphi}\right\rangle$. Now our task is to commute all the annihilation operators in $c(\sigma)$ through the creation operators in $\left|I^{\varphi}\right\rangle$. This is a simple but slightly tedious exercise in using the following operator identities

$$
e^{A} e^{B}=e^{B} e^{A} e^{[A, B]}, \quad e^{A} e^{B}=e^{A+B} e^{\frac{1}{2}[A, B]}
$$

and

$$
e^{\frac{1}{4} \vec{O} \omega \vec{O}}=\pi^{-N / 2}\left(\operatorname{det} \omega^{-1}\right)^{(1 / 2)} \int D \vec{X} e^{-\vec{X} \omega^{-1} \vec{X}} e^{\vec{X} . \vec{O}}
$$

Hence $^{11}$

$$
c(\sigma)\left|I^{\varphi}>=e^{i \phi\left(\frac{\pi}{2}\right)\left(1-\frac{3}{2} y\right)} e^{i k(\sigma)} e^{-\gamma_{n}^{(r)}(\sigma) b_{-n}^{(r)}}\right| I_{0}^{\varphi}>
$$

where

$$
\begin{gathered}
k(\sigma)=-i\left\{-\frac{2}{\pi} \sum_{n=1}^{\infty} \frac{1}{(2 n-1)^{3}}+i \frac{\sigma}{2}(1-3 y)-\frac{3}{2} i y \sum_{n=1}^{\infty} \frac{(-)^{n} \sin n(2 \sigma)}{n}\right. \\
\left.+D+D^{*}+C+\frac{2}{\pi^{2}} \sum_{n=1}^{\infty} \frac{1}{(2 n-1)} \partial_{\sigma, n}^{*} \Sigma_{n}^{c}(\sigma)-\sum_{n=1}^{\infty} \alpha_{n}^{(1)} \alpha_{n}^{(2)}\right\} \\
\gamma_{n}^{(1)}(\sigma)=\gamma_{n}^{(2)^{*}}(\sigma)=\alpha_{n}^{(1) *}+\alpha_{n}^{(2)}
\end{gathered}
$$

and $\left|I_{0}^{\varphi}\right\rangle$ is the exponential of the quadratic form in ghost creation operators (see (1)). The operator part in (17) is even under $\sigma \longrightarrow \pi-\sigma$ for all $\sigma$ including the mid-point. This is easily seen by replacing $\sigma$ by $\pi-\sigma$ in the explicit expression for $\gamma_{n}^{(r)}(\sigma)$. This result agrees with the standard result for the FS $[1,16]$. For the phase factor we have first to evaluate the various quantities and sums in the expression for $k(\sigma)$. These sums are potentially divergent and have to be treated with care. Here we only give the final results, the technical details are given at the end of the paper. Hence

\footnotetext{
${ }^{11}$ We have put a factor $y$ in the ghost insertion in the identity operator to emphasize the importance of the coefficient $-\frac{3}{2}$.
} 


$$
\begin{gathered}
k(\sigma)= \begin{cases}0, & \text { if } \quad|\sigma|<\frac{\pi}{2} \\
-\pi, & \text { if } \frac{\pi}{2}<\sigma<\pi \\
\pi, & \text { if } \quad-\pi<\sigma<-\frac{\pi}{2}\end{cases} \\
k\left(\frac{\pi}{2}\right)=-\frac{\pi}{2}
\end{gathered}
$$

This means that $e^{i k(\sigma)}$ is odd under $\sigma \longrightarrow \pi-\sigma$ except at $\sigma=\frac{\pi}{2}$ as expected. We notice that this result has been obtained in the original work of Witten [1]. This completes the proof of the $c$-overlaps for the identity vertex. It remains to show that the $b$-overlaps are satisfied as well. Repeating the same procedure, for the $b$-overlaps, we obtain for the phase factor (we call it $e^{i \tilde{k}(\sigma)}$ )

$$
\begin{aligned}
& \tilde{k}(\sigma)=\left\{\begin{array}{l}
\sigma, \quad \text { if } \quad|\sigma|<\frac{\pi}{2} \\
-2 \pi+\sigma, \quad \text { if } \frac{\pi}{2}<\sigma<\pi \\
2 \pi+\sigma, \quad \text { if } \quad-\pi<\sigma<-\frac{\pi}{2}
\end{array}\right. \\
& \tilde{k}\left(\frac{\pi}{2}\right)=-\frac{\pi}{2}
\end{aligned}
$$

which means that $e^{i \tilde{k}(\sigma)}$ is even under $\sigma \longrightarrow \pi-\sigma$ including $\sigma=\frac{\pi}{2}$ as expected. This is again the standard result obtained in the literature. With this we conclude the proof for the two realization of the identity vertex.

\section{The 3-Vertex}

We now turn to the question of the 3-Vertex. However, we find it more instructive to consider a general vertex of order $N\left(V_{N}\right)$. The reason for this is that discussing a general vertex makes manifest the fact that higher vertices (i.e. $N>3$ ) can be excluded from the theory without having to impose BRST invariance by using only the overlap equations defining the ghost vertex and ghost counting $^{12}$. It has been shown in [1] that BRST invariance requires the insertion factor of $e^{ \pm \frac{3}{2} i \phi\left(\frac{\pi}{2}\right)}$ at the string mid-point . To include higher vertices $(N>3)$ in WSFT we need to change the ghost insertions at the string midpoint, if we change the insertion factor to construct a BRST invariant $N$-Vertex $(N>3)$ the ghost number counting comes out wrong $[1,12,13]$. Furthermore, changing the ghost insertions to cure this problem breaks in turn the BRST

\footnotetext{
${ }^{12}$ One has to remember that these two conditions are necessary for the proof of the BRST invariance.
} 
invariance (for more details on this point we refer the reader to the following references $[1,12,13])$.

To establish the bose-fermi equivalence of the two versions of the vertex we have to show that the bose realization of the $N$-Vertex, (1) satisfy the $c$ and $b$ overlap conditions given in (9) and (10). Recall

$$
\left|V_{N}^{\varphi}>=e^{i g_{\mu} \sum_{\mu=1}^{N} \phi_{\mu}\left(\frac{\pi}{2}\right)}\right| V_{N, 0}^{\varphi}>
$$

where $g_{\mu}$ is the ghost number insertion at the mid-point of the string to be specified later ${ }^{13}$. The action of $c_{\mu}(\sigma)$ on the above vertex gives

$$
c_{\mu}(\sigma)\left|V_{N}^{\varphi}>=e^{i \phi_{\mu}\left(\frac{\pi}{2}\right)\left(1+g_{\mu}\right)} e^{i k_{\mu}(\sigma)} e^{(\text {creation operators })}\right| V_{N, 0}^{\varphi}>
$$

where

$$
\begin{aligned}
k_{\mu}(\sigma)= & -i\left\{-\frac{2}{\pi} \sum_{n=1}^{\infty} \frac{1}{(2 n-1)^{3}}+i \frac{\sigma}{2}\left(1+2 g_{\mu}\right)+2 i g_{\mu} \sum_{n=1}^{\infty} \frac{(-)^{n} \sin n(2 \sigma)}{n}\right. \\
& \left.+D+D^{*}+C+\frac{2}{\pi^{2}} \sum_{n=1}^{\infty} \frac{1}{(2 n-1)} \partial_{\sigma, n}^{*} \Sigma_{n}^{c}(\sigma)-\frac{1}{2} \vec{\xi}^{T} \Theta \vec{\xi}\right\}
\end{aligned}
$$

(with $\xi_{n \nu}^{r}(\mu)=\alpha_{n}^{r} \delta_{\mu \nu}$ ). The operator part of $c$-overlaps is satisfied for all $\sigma$ as noted earlier. It is not hard to show that $\vec{\xi}^{T} \Theta \vec{\xi}=0$. Evaluating the remaining terms in the above expression (as we have done for the case of the identity, see appendix) we have

$$
\begin{gathered}
k_{\mu}(\sigma)=0, \text { if }|\sigma|<\frac{\pi}{2}, \\
k_{\mu-1}(\pi-\sigma)=\frac{\pi}{2}\left(1+2 g_{\mu}\right), \text { if }|\sigma|<\frac{\pi}{2} \\
k_{\mu-1}(\sigma-\pi)=-\frac{\pi}{2}\left(1+2 g_{\mu}\right), \text { if }|\sigma|<\frac{\pi}{2} \\
k_{\mu}\left(\frac{\pi}{2}\right)=k_{\mu-1}\left(\frac{\pi}{2}\right)=\frac{\pi}{4}\left(1+2 g_{\mu}\right)-\frac{i}{2} \sum_{n=1}^{\infty} \frac{1}{2 n}
\end{gathered}
$$

\footnotetext{
${ }^{13} \mathrm{~g}$ has to be chosen to ensure that the overlap equations are satisfied and the ghost counting is correct. It was shown in $[1,9,10,13,6]$ that BRST invariance restrict severly the choice of $\mathrm{g}$.
} 
Note that the above results are independent of the string index $\mu$. To satisfy the overlap equations in (9) the phase factor has to satisfy

$$
e^{i k_{\mu}(\sigma)}=-e^{i k_{\mu-1}(\pi-\sigma)}, \text { for } \mu=1,2, \ldots, N
$$

We see that the above condition is true for

$$
g_{\mu}=2 n-\frac{3}{2}, \mu=1,2,3, \ldots, N
$$

(n integer) except at the point $\sigma=\frac{\pi}{2}$ as expected. In the case of interest (i.e. $N=3$ ), $g_{\mu}=\frac{1}{2}$ which amounts to $n=1$. Thus we have shown that the bosonized realization of the ghost 3 -Vertex obey the $c$-overlaps, except at the mid-point, as expected. To complete the proof we need to show that the $b$-overlap equations (10) are also satisfied by the bosonized realization of the vertex. Following similar steps to those employed for the $c$ part we discover that the $b$ overlap equations hold as well for all values of $\sigma$, including the point $\sigma=\frac{\pi}{2}$, as expected.

\section{Conclusions}

It is satisfying to see that (21) depends on the ghost insertion $e^{i g_{\alpha} \phi_{\alpha}\left(\frac{\pi}{2}\right)}$. This was established in the original work of Witten on interacting string field theory [1]. Now the interesting thing here is that the above formula is true for a general vertex of order $N$ which enables us to discuss the points we raised earlier regarding BRST invariance and higher vertices $(N>3)$. If we consider $N=4$, the ghost number is given through the relation $V_{3}^{\varphi}=I^{\varphi} V_{4}^{\varphi}$. Recalling the fact ${ }^{14}$ that $I^{\varphi}$ changes the ghost number by $-\frac{3}{2}$ and $V_{3}^{\varphi}$ has a ghost number equal to $\frac{3}{2}$, we deduce that the natural ghost number for $V_{4}$ is 3 and therefore $g=\frac{3}{4}$ (where we have made use of the fact ${ }^{15}$ that $\left.\phi_{1}\left(\frac{\pi}{2}\right)=\phi_{2}\left(\frac{\pi}{2}\right)=\phi_{3}\left(\frac{\pi}{2}\right)=\phi_{4}\left(\frac{\pi}{2}\right)\right)$. Now the point to observe is that this value of $g$ is not a solution of (21) which amounts to the violation of the overlap equations for $c$ for all values of $\sigma$. Repeating the procedure for other vertices $(N>4)$ we see that eq. $(21)$ is always violated if the ghost counting is correct (i.e. deduced from the fact $V_{N-1}=I V_{N}$ ). In conclusion all the ghost number values given by the above formula, which satisfy the overlap equations, by virtue of the derivation, do indeed spoil the ghost number counting for all $\mathrm{n}$ (integer) apart from $n=0$ (identity vertex) and $n=1$ (3-Vertex). Changing the insertion to cure this problem violates in turn the overlap equations. Therefore the important point to note here is that we

\footnotetext{
${ }^{14} \mathrm{~A}$ derivation of this statement has been given in [1].

${ }^{15}$ this is the case because of delta functions in the definition of the vertex.
} 
did not need to impose either the $K_{n}$ or the BRST (Q) symmetry to exclude higher vertices ${ }^{16}$. Hence we see in the HS formulation of interacting string field theory, the only possible vertex is the 3 -Vertex and all higher $(N>3)$ vertices are excluded by the requirement that both the overlap equations (defining the interaction vertices in the theory) and ghost number counting are satisfied. This result was precisely established in the work of Witten [1]. The fact that we can establish this conclusion, using the HS operator formulation of WSFT, for all $N=1,2,3, \ldots, \infty$ (unlike in the case of the full string operator formulation of $[12,10,13]$ where only up to $N=4$ vertices were constructed), is not only satisfying, but makes manifest the calculating power of the HS operator approach to interacting string field theory.

An interesting question arises. In closed string field theory the vertices are constructed as the product of two open string vertices, one for the left and one for the right moving modes. For the orbital degrees of freedom this was seen to be a consequence of the string overlaps defining the vertex in reference [7]. In the light of the results found in this paper, it is interesting to see if the bosefermi equivalence has any implications when one includes the ghost degrees of freedom in formulating the field theory of closed strings. This is currently under investigation.

\section{Appendix}

In this appendix we give the steps in evaluating $k(\sigma)$. Recall that

$$
\begin{aligned}
k(\sigma) & =-i\left\{-\frac{2}{\pi} \sum_{n=1}^{\infty} \frac{1}{(2 n-1)^{3}}+i \frac{\sigma}{2}(1-3 y)-\frac{3}{2} i y \sum_{n=1}^{\infty} \frac{(-)^{n} \sin n(2 \sigma)}{n}\right. \\
& \left.+D+D^{*}+C+\frac{2}{\pi^{2}} \sum_{n=1}^{\infty} \frac{1}{(2 n-1)} \partial_{\sigma, n}^{*} \Sigma_{n}^{c}(\sigma)-\sum_{n=1}^{\infty} \alpha_{n}^{(1)} \alpha_{n}^{(2)}\right\}
\end{aligned}
$$

To evaluate the above expression, first, consider

$$
D+D^{*}+C=2 \operatorname{Re}(D)+C
$$

Substituting the explicit expressions for $D$ and $C$ and then making use of the following identity,

\footnotetext{
${ }^{16}$ It was shown in ref. $[1,9,10,13,6]$ that changing the insertion to fix this problem breaks both $K_{n}$ and $Q$ (BRST) symmetry, since the phase factors contribute critically to the mid-point anomalies.
} 


$$
\sum_{m=1}^{\infty} \frac{2 n}{2 m-1} B_{2 n 2 m-1} B_{2 m-12 k}=-\frac{1}{4} \delta_{n k}
$$

the above expression reduces to

$$
\begin{aligned}
D+D^{*}+C & =\frac{1}{2} \sum_{n=1}^{\infty} \frac{1}{2 n}-\frac{1}{2 \pi^{2}} \sum_{n=1}^{\infty}(2 n-1)\left(\Sigma_{n}^{c}(\sigma)\right)^{2} \\
& -\frac{2}{\pi^{2}} \sum_{n=1}^{\infty} \frac{1}{2 n-1}\left(\Sigma_{n}^{s}(\sigma)\right)^{2}
\end{aligned}
$$

where

$$
\Sigma_{n}^{s}(\sigma)=\sum_{m=1}^{\infty} \frac{m(-)^{m} \sin m(2 \sigma)}{m^{2}-\left(\frac{2 n-1}{2}\right)^{2}}
$$

The sums in the above expression are potentially divergent and have to be handled with care, however there is no need to evaluate all of them since there are contributions with opposite signs coming from the other terms in (22) as we shall see bellow. The $\alpha_{n}^{(1)} \alpha_{n}^{(2)}$ term gives

$$
\begin{gathered}
\sum_{n=1}^{\infty} \alpha_{n}^{(1)} \alpha_{n}^{(2)}=\frac{2}{\pi^{2}} \sum_{n=1}^{\infty} \frac{1}{(2 n-1)^{3}}-\frac{2}{\pi^{2}} \sum_{n=1}^{\infty} \frac{1}{(2 n-1)} \partial_{\sigma, n} \Sigma_{n}^{c}(\sigma) \\
+\frac{1}{2 \pi^{2}} \sum_{n=1}^{\infty}(2 n-1)\left(\partial_{\sigma, n} \Sigma_{n}^{c}(\sigma)\right)^{2}-\frac{1}{2} \sum_{n=1}^{\infty} \frac{1}{2 n-1} e^{-2 i(2 n-1) \sigma}
\end{gathered}
$$

where

$$
\left(\partial_{\sigma, n} \Sigma_{n}^{c}(\sigma)\right)^{2}=\left(\Sigma_{n}^{c}(\sigma)\right)^{2}+\frac{2 i}{(2 n-1)} \Sigma_{n}^{c}(\sigma) \partial_{\sigma} \Sigma_{n}^{c}(\sigma)-\frac{1}{(2 n-1)^{2}}\left(\partial_{\sigma} \Sigma_{n}^{c}(\sigma)\right)^{2}
$$

Putting everything together we arrive at

$$
\begin{gathered}
k(\sigma)=\frac{\sigma}{2}(1-3 y)-\frac{3}{2} y \sum_{n=1}^{\infty} \frac{(-)^{n} \sin n(2 \sigma)}{n}+\frac{4 i}{\pi^{2}} \sum_{n=1}^{\infty} \frac{1}{(2 n-1)^{3}}-\frac{i}{2} \sum_{n=1}^{\infty} \frac{1}{2 n} \\
-\frac{4 i}{\pi^{2}} \sum_{n=1}^{\infty} \frac{1}{(2 n-1)} \Sigma_{n}^{c}(\sigma)+\frac{i}{\pi^{2}} \sum_{n=1}^{\infty}(2 n-1)\left(\Sigma_{n}^{c}(\sigma)\right)^{2}+\frac{2}{\pi^{2}} \sum_{n=1}^{\infty} \Sigma_{n}^{c}(\sigma) \Sigma_{n}^{s}(\sigma) \\
-\frac{i}{2} \sum_{n=1}^{\infty} \frac{\cos (2 n-1)(2 \sigma)}{2 n-1}+\frac{1}{2} \sum_{n=1}^{\infty} \frac{\sin (2 n-1)(2 \sigma)}{2 n-1}
\end{gathered}
$$


In performing the remaining sums in the above expression we have to specify the range of $\sigma$ as those sums will have different analytical expressions for different ranges in $\sigma$. Making use of

eq. (23) reduces to

$$
\begin{gathered}
\Sigma_{n}^{c}(\sigma)=\frac{2}{(2 n-1)^{2}}+\frac{\pi(-)^{n}}{2 n-1} \cos (2 n-1) \sigma, \text { if } \quad|\sigma| \leq \frac{\pi}{2}, \\
\Sigma_{n}^{s}(\sigma)=\frac{\pi(-)^{n}}{2} \sin (2 n-1) \sigma, \text { if } \quad|\sigma|<\frac{\pi}{2}
\end{gathered}
$$

$$
k(\sigma)=-\frac{i}{2} \sum_{n=1}^{\infty} \frac{1}{2 n}+i \sum_{n=1}^{\infty} \frac{\cos ^{2}(2 n-1) \sigma}{2 n-1}-\frac{i}{2} \sum_{n=1}^{\infty} \frac{\cos (2 n-1)(2 \sigma)}{2 n-1}, \text { if }|\sigma|<\frac{\pi}{2}
$$

where we have made use of

$$
\sum_{n=0}^{\infty} \frac{(-)^{n+1} \sin (2 n+1) \sigma}{(2 n+1)^{2}}=-\frac{\pi}{4} \sigma, \text { if } \quad|\sigma| \leq \frac{\pi}{2}
$$

Making use of the trig. identity $\cos 2 A=2 \cos ^{2} A-1$ we obtain

$$
k(\sigma)=0, \text { if } \quad|\sigma|<\frac{\pi}{2}
$$

letting $\sigma \longrightarrow \pi-\sigma$ in (23) and going through similar steps as above we arrive at

$$
k(\pi-\sigma)=\frac{\pi}{2}(1-3 y), \text { if } \quad|\sigma|<\frac{\pi}{2} .
$$

For $\sigma \longrightarrow \sigma-\pi,(23)$, gives

$$
k(\sigma-\pi)=-\frac{\pi}{2}(1-3 y), \text { if } \quad|\sigma|<\frac{\pi}{2}
$$

It remains to evaluate $k(\sigma)$ at the mid-point. Thus setting $\sigma=\frac{\pi}{2}$ in (23) we get

$$
k\left(\frac{\pi}{2}\right)=-\frac{\pi}{4}(1-3 y)
$$

Setting $y=1$ in (25) and (26) and combining eqs. (24), (25), (26) and (27) we arrive at eq.(18).

The work of A.A. has been supported by the SSR, Tripoli, Libya. The work of J.B. has been supported by CICYT under grant number 93-0234 . 


\section{References}

[1] E. Witten, Nucl. Phys. B268 (1986) 253.

[2] J.L. Gervais, Nucl. Phys. B276 (1986) 253.

[3] H. M. Chan and S.T. Tsou, Phys. Rev. D35 (1987) 2474; D39 (1989) 555.

[4] J. Bordes, H.M. Chan, L. Nellen and S.T. Tsou, Nucl. Phys. B351 (1991) 441

J. Bordes, A. Abdurrahman and F. Anton, Phys. Rev. D49 (1994) 2966.

[5] A. Abdurrahman, F. Anton and J. Bordes, Nucl. Phys. B397 (1993) 260.

[6] A. Abdurrahman, F. Anton and J. Bordes, Nucl. Phys. B411 (1994) 693.

[7] H. M. Chan, J. Bordes, S.T. Tsou and L. Nellen, Phys. Rev. D44 (1991) 1786 ;

F. Anton, A. Abdurrahman and J. Bordes, Phys. Lett. B327 (1994) 234;

F. Anton, A. Abdurrahman and J. Bordes Phys. Lett B333 (1994) 337;

F. Anton, A. Abdurrahman and J. Bordes, Rutherford Appleton Laboratory preprint RAL-94-065.

[8] T. Mo

rris, Nucl. Phys. B297 (1988) 141;

R. Potting, C. Taylor and B. Velikson, Phys. Lett. B198 (1987) 184;

J. L. Manes, Nucl. Phys. B303 (1988) 305;

R.Potting and C. Taylor, Nucl. Phys. B316 (1989) 59;

M. Maens, Osaka University preprint OU-HET-136.

[9] D. Gross and A. Jevicki, Nucl. Phys. B287 (1987) 225.

[10] K. Itoh, K. Ogawa and K. Suehiro, Nucl. Phys. B289 (1987) 127.

[11] E. Cremmer, A Schwimmer and C. Thorn, Phys. Lett. B179 (1986) 57;

S. Samuel, Phys. Lett. B181 (1986) 225.

[12] D. Gross and A. Jevicki, Nucl. Phys. B283 (1987) 1.

[13] A. Jevicki, Int. J. Mod. Phys. A3 (1988) 299.

[14] S. Mandelstam, Nucl. Phys. B64 (1973) 209. 
[15] W. Siegel and B. Zwiebach, Nucl. Phys. B263 (1986) 105.

[16] Z. Hlousek and A. Jevicki, Nucl. Phys. B288 (1987) 131. 